\title{
Stability of fold maps of manifolds with boundary
}

\author{
Naoki SHibata \\ (Received March 14, 2001; Revised June 10, 2002)
}

\begin{abstract}
Let $X$ be a compact orientable smooth $m$-manifold with non-empty boundary, $Y$ a smooth $n$-manifold $(m \geq n)$ and $f: X \rightarrow Y$ a stable map with only fold singularities. In this paper we clarify the structure of the fold set of the stable map $f$ near the boundary.
\end{abstract}

Key words: smooth manifold, boundary, stable map, singularity, fold point.

\section{Introduction}

In the previous paper Shibata [7], we considered the non-singular stable maps of compact orientable 3-manifolds with non-empty boundary into $\mathbf{R}^{2}$. In history, $\mathrm{H}$. Levine [4] studied the stable maps of compact $n$-dimensional manifolds $(n \geq 2)$ with non-empty boundary into $\mathbf{R}^{2}$ under the condition that each singular set does not intersect with the boundary. However, as will be explained in this paper, the notion of stability is also effective for the case that each singular set is possible to intersect with the boundary. In fact, we will clarify how the singular set intersects with the boundary in the case of fold stable maps.

In this paper, the domain manifold $X$ is a compact orientable smooth $m$-manifold with non-empty boundary and $Y$ is a smooth $n$-manifold, and the stable maps $X \rightarrow Y$ will have singularities of fold points only, which are defined in $\S 2$.

In $\S 3$, we prove Lemma 6 which will be used for the characterization of fold stable maps on manifolds with boundary in $\S 4$. Moreover in $\S 3$, we prove Proposition 1 which characterizes the stability of immersions on manifolds with boundary. Proposition 1 explains that the fold stable maps restricted on the set of fold points are also stable based on Theorem 1 in $\S 4$.

In $\S 4$, we prove our main result as Theorem 1 which clarifies how the singularities of fold stable maps $X \rightarrow Y(m \geq n)$ intersect with the bound- 
ary. The method of proof belongs to geometric topology rather than the theory of singularity. In the proof of Theorem 1, we give a quasi-normal form of fold singularities around the boundary as Lemma 7. When $X$ is a closed manifold, the corresponding result has been proven in GolubitskyGuillemin [3]. However for our case with boundary, we need some original techniques different from the case without boundary.

\section{Preliminaries}

Let $X$ be a $m$-dimensional smooth manifold with or without boundary and $Y$ a smooth $n$-manifold. We denote by $C^{\infty}(X, Y)$ the set of the smooth maps of $X$ into $Y$ with the Whitney $C^{\infty}$ topology. First, we define $d f$ at $p \in \partial X$ or at $f(p) \in \partial Y$. For $f: X \rightarrow Y$, we extend $f$ slightly to a smooth map $\tilde{f}: \widetilde{X} \rightarrow \tilde{Y}$ which satisfies $\tilde{f} \mid X=f$ where $\widetilde{X} \supset X$ and $\tilde{Y} \supset Y$ are open manifolds. Then we regard $T_{p} \widetilde{X}$ and $T_{f(p)} \widetilde{Y}$ as the tangent spaces at $p \in \partial X$ and $f(p) \in \partial Y$ respectively. Therefore, the rank of $d f$ at $p \in \partial X$ is different from that of $d(f \mid \partial X)$ at $p$. For a smooth map $f: X \rightarrow Y, S(f)$ denotes the singular set of $f$; i.e., $S(f)$ is the set of the points in $X$ where the rank of the differential $d f$ is strictly less than $\min \{m, n\}$. A smooth map $f: X \rightarrow Y$ is stable if there exists an open neighborhood $N(f)$ of $f$ in $C^{\infty}(X, Y)$ such that every $g$ in $N(f)$ is right-left equivalent to $f$; i.e., there exist diffeomorphisms $\phi: X \rightarrow X$ and $\varphi: Y \rightarrow Y$ satisfying $g=\varphi \circ f \circ \phi^{-1}$.

Next, we give a definition of the fold point for the case with boundary. We can define $J^{1}(X, Y)$ in the usual way, which has a structure of a manifold. For $\sigma \in J^{1}(X, Y)$, let $h$ be a representative of $\sigma$ in $C^{\infty}(X, Y)$ and put corank $\sigma=\min (\operatorname{dim} X, \operatorname{dim} Y)-\operatorname{rank}(d h)_{p}$, where $d h$ is already defined. Then define $S_{r}=\left\{\sigma \in J^{1}(X, Y) \mid \operatorname{corank} \sigma=r\right\}$, and for a stable map $f: X \rightarrow Y$, define $S_{1}(f)=\left(j^{1} f\right)^{-1}\left(S_{1}\right)$.

Definition 1 For $p \in S_{1}(f), p$ is also in $S_{1,0}(f)$ if the following (i) or (ii) is satisfied.

(i) When $p$ is in Int $X, j_{1} f$ is transverse to $S_{1}$ at $p$ and $T_{p} S_{1}(f)+$ $\operatorname{Ker}(d f)_{p}=T_{p} X$ is satisfied.

(ii) When $p$ is in $\partial X$, we extend $f$ around $p$ to $\tilde{f}$ which is a smooth map from an extended manifold $\widetilde{X} \supset X$ to an extended manifold $\widetilde{Y} \supset Y$ so that $p \in \operatorname{Int} \widetilde{X}$ and $\tilde{f} \mid X=f$ are satisfied. Then $p \in S_{1}(\tilde{f})$ satisfies that $j_{1} \tilde{f}$ is transverse to $S_{1}$ at $p$ and that $T_{p} S_{1}(\tilde{f})+\operatorname{Ker}(d \tilde{f})_{p}=T_{p} \tilde{X}$.

In addition, if $p \in S_{1,0}(f)$ satisfies (i) or (ii) with the condition that 
$S_{1}(\tilde{f})$ is transverse to $\partial X$ at $p$, then $p$ is said to be a fold point.

Definition 2 We call $f: X \rightarrow Y$ a submersion with $S_{1,0}$ (or folds) if $S(f)=S_{1,0}(f)($ resp. $=\{$ folds $\})$.

\section{Stability of immersions on manifolds with boundary}

In this section, we prepare several lemmas to treat the stability of smooth maps on manifolds with boundary. In addition, we characterize the stability of immersions on manifolds with boundary.

Let $X$ be a compact orientable smooth $m$-manifold with boundary and $Y$ a smooth $n$-manifold. Then we define an $\left(s, s^{\prime}\right)$-fold multi- $k$-jet bundle $J_{s, s^{\prime}}^{k}(X, Y)$. Let $X^{s, s^{\prime}}=\underbrace{X \times \cdots \times X}_{s} \times \underbrace{\partial X \times \cdots \times \partial X}_{s^{\prime}}$ and $X^{\left(s, s^{\prime}\right)}=$ $\left\{\left(x_{1}, \ldots, x_{s}, x_{s+1}, \ldots, x_{s+s^{\prime}}\right) \in X^{s, s^{\prime}} \mid x_{i} \neq x_{j}\right.$ for $\left.1 \leq i<j \leq s+s^{\prime}\right\}$. For $\alpha: J^{k}(X, Y) \rightarrow X$ the source map, we define

$$
\begin{aligned}
\alpha^{s, s^{\prime}}: J^{k}(X, Y)^{s, s^{\prime}} & \\
& =\underbrace{J^{k}(X, Y) \times \cdots \times J^{k}(X, Y)}_{s} \times \underbrace{J^{k}(\partial X, Y) \times \cdots \times J^{k}(\partial X, Y)}_{s^{\prime}} \rightarrow X^{s, s^{\prime}}
\end{aligned}
$$

as the induced map by $\alpha$. Then

$$
J_{s, s^{\prime}}^{k}(X, Y)=\left(\alpha^{s, s^{\prime}}\right)^{-1}\left(X^{\left(s, s^{\prime}\right)}\right)
$$

is called an $\left(s, s^{\prime}\right)$-fold multi-k-jet bundle.

$X^{\left(s, s^{\prime}\right)}$ is a manifold since it is an open subset of $X^{s, s^{\prime}}$. Thus $J_{s, s^{\prime}}^{k}(X, Y)$ is an open subset of $J^{k}(X, Y)^{s, s^{\prime}}$ and is also a smooth manifold.

Let $f: X \rightarrow Y$ be smooth. Then we can also define $j_{s, s^{\prime}}^{k} f: X^{\left(s, s^{\prime}\right)} \rightarrow$ $J_{s, s^{\prime}}^{k}(X, Y)$ by

$$
\begin{aligned}
& j_{s, s^{\prime}}^{k} f\left(x_{1}, \ldots, x_{s+s^{\prime}}\right) \\
& \quad=\left(j^{k} f\left(x_{1}\right), \ldots, j^{k} f\left(x_{s}\right), j^{k}(f \mid \partial X)\left(x_{s+1}\right), \ldots, j^{k}(f \mid \partial X)\left(x_{s+s^{\prime}}\right)\right) .
\end{aligned}
$$

Lemma 1 (Multijet Transversality Theorem; the case with boundary) Let $W \subset J_{s, s^{\prime}}^{k}(X, Y)$ be a submanifold. Then $T_{w}=\left\{f \in C^{\infty}(X, Y) \mid j_{s, s^{\prime}}^{k} f\right.$ is transverse to $W\}$ is a residual subset of $C^{\infty}(X, Y)$.

Proof. It is sufficient to modify the proof of Theorem 4.13 in [3, p. 57] for the case with boundary. The concept of jets is local, and local arguments of jets on closed manifolds hold on manifolds with boundary. Since almost 
all needed lemmas depend on the local arguments of jets, they are easily modified relatively for our proof.

Lemma 2 (Whitney Immersion Theorem; the case with boundary) Suppose that $X$ and $Y$ satisfy $\operatorname{dim} Y \geq 2 \cdot \operatorname{dim} X$. Then the set of immersions $X \rightarrow Y$ is an open dense subset of $C^{\infty}(X, Y)$ in the Whitney $C^{\infty}$ topology.

Proof. On the proof of Theorem 5.6 in [3, p. 61], use the modified version of Thom transversality theorem for the case with boundary.

Lemma $3 C^{\infty}(X, Y)$ is a Baire space in the Whitney $C^{\infty}$ topology.

Proof. It is similar with the case without boundary.

Definition 3 Let $f: X \rightarrow Y$ be a smooth map and $f^{\left(s, s^{\prime}\right)}: X^{\left(s, s^{\prime}\right)} \rightarrow$ $Y^{s+s^{\prime}}$ the restriction of $\underbrace{f \times \cdots \times f}_{s} \times \underbrace{(f \mid \partial X) \times \cdots \times(f \mid \partial X)}_{s^{\prime}}: X^{s, s^{\prime}} \rightarrow$ $Y^{s+s^{\prime}}$ to $X^{\left(s, s^{\prime}\right)}$. Then $f$ is a mapping with strictly normal crossings if for every $s, s^{\prime}\left(s+s^{\prime}>1\right), f^{\left(s, s^{\prime}\right)}$ is transverse to $\Delta Y^{s+s^{\prime}}=\{(y, \ldots, y) \in$ $\left.Y^{s+s^{\prime}} \mid y \in Y\right\}$.

Lemma 4 The set of mappings of $X$ into $Y$ with only strictly normal crossings is dense in $C^{\infty}(X, Y)$.

Proof. Use the arguments of Proposition 3.2 in [3, p. 82] with the notion of strictly normal crossings. In addition, use Lemma 1 and Lemma 3 for the case with boundary instead of the corresponding facts for the case without boundary.

Corollary 1 Immersions with strictly normal crossings are dense in the set of all immersions $X \rightarrow Y$.

Lemma 5 If $f: X \rightarrow Y$ is an immersion which is stable, then $f$ is an immersion with strictly normal crossings and $f(X) \subset \operatorname{Int} Y$ is satisfied.

Proof. If $f(X) \cap \partial Y \neq \emptyset$ is satisfied then a slight perturbation of $f$ is possible to change $f$ such as $f(X) \cap \partial Y=\emptyset$, however this perturbation is not expressed by the right-left equivalence. This indicates the latter claim.

Moreover, any mapping equivalent to an immersion with strictly normal crossings is an immersion with strictly normal crossings, thus Lemma 4 implies the former claim. 
To treat manifolds with boundary, we recall a definition in Shibata [7], and in addition, we prepare a useful lemma as follows.

Definition 4 Let $\alpha: X \rightarrow Y$ be a smooth map and $\pi_{Y}: T Y \rightarrow Y$ the canonical projection. A smooth map $w: X \rightarrow T Y$ is called a vector field along $\alpha$ if $w$ satisfies $\alpha=\pi_{Y} \circ w$. Then we say that $\alpha$ is strongly infinitesimally stable if for every $w$, a vector field along $\alpha$, there always exist a vector field $s$ on $X$ whose restriction to $\partial X$ is a vector field on $\partial X$ (i.e., each vector of $s$ on $\partial X$ is tangent to $\partial X$ ) and a vector field $t$ on $Y$ whose restriction to $\partial Y$ is a vector field on $\partial Y$ such that

$$
w=(d \alpha) \circ s+t \circ \alpha,
$$

where $d \alpha: T X \rightarrow T Y$ is the differential of $\alpha$.

Henceforth, we denote the set of vector fields along $\alpha: X \rightarrow Y$ by $C_{\alpha}^{\infty}(X, T Y)$.

Lemma 6 A smooth map $f: X \rightarrow Y$ is stable if and only if $f$ is strongly infinitesimally stable.

Outline of proof. Necessity is obtained by modifying the argument in [3]. To begin with, we define the notion of strongly transverse stable as follows: Let $\sigma$ be an $(s, 0)$-fold multijet in $J_{s, 0}^{k}(X, Y)$, then, let $\mathcal{D}_{\sigma}^{s}$ be the orbit through $\sigma$ under the action of $\operatorname{Diff}(X) \times \operatorname{Diff}(Y)$ on $J_{s, 0}^{k}(X, Y)$. We can prove that $\mathcal{D}_{\sigma}^{s}$ is an immersed submanifold without boundary of $J_{s, 0}^{k}(X, Y)$ for each $\sigma$. Furthermore, $\mathcal{D}_{\sigma}^{s}$ is a submanifold without boundary in $J_{s, 0}^{k}(X, Y)$. Then $f$ is said to be strongly transverse stable if for every $s$ with $1 \leq s \leq$ $n+1$ and diagonal element $\sigma$ in $J_{s, 0}^{n}(X, Y), j_{s, 0}^{n} f$ is transverse to $\mathcal{D}_{\sigma}^{s}$.

First, we show that, if $f$ is stable then $f$ is strongly transverse stable. Second, we show that, if a smooth map $X \rightarrow Y$ is strongly transverse stable with $f(X) \subset \operatorname{Int} Y$, then $f$ is strongly infinitesimally stable. In this process of our proof, we need to use the notation $C^{\infty}(T X)_{p}(p \in X)$ in the sense that $C^{\infty}(T X)$ denotes the set of vector fields on $T X$ whose restrictions onto $\partial X$ are vector fields on $\partial X$; The same for $Y$. Details of these modifications are not difficult.

Sufficiency has been shown by von Essen [2] based on Mather's theory [5]. We can remark that the above lemma is a special case of the generalized theorem which is obtained in Shibata [6]. 
Corollary 2 If $f: X \rightarrow Y$ is stable, then $f \mid \partial X$ is also stable.

Proof. Let $w$ be a vector field along $f \mid \partial X$, and let $\bar{w}$ be a vector field along $f$ which is an arbitrary extension of $w$. Then by Lemma 6 , $\bar{w}$ is expressed by $\bar{w}=(d f) \circ s+t \circ f$ where $s$ is a vector field on $X$ tangential to $\partial X$ on $\partial X$ and $t$ is a vector field on $Y$ tangential to $\partial Y$ on $\partial Y$. So, $w=\bar{w} \mid \partial X=d(f \mid \partial X) \circ(s \mid \partial X)+t \circ(f \mid \partial X)$ is satisfied. Hence, if $f$ is stable then $f \mid \partial X$ is stable.

Definition 5 Let $V=\mathbf{R} \cdot v_{1}+\cdots+\mathbf{R} \cdot v_{k}$ be a $k$-dimensional vector space in $\mathbf{R}^{l}$ with a fixed basis $v_{i}(1 \leq i \leq k \leq l)$. Then $V^{\partial}$ is defined by $\left\{\alpha_{1} \cdot v_{1}+\alpha_{2} \cdot v_{2}+\cdots+\alpha_{k} \cdot v_{k} \mid \alpha_{1} \in \mathbf{R}_{\geq 0}, \alpha_{i} \in \mathbf{R}\right.$ for $\left.i \neq 1\right\}$.

Proposition 1 Let $f: X \rightarrow Y$ be an immersion. Then $f$ is stable if and only if $f(X) \subset$ Int $Y$ and $f$ has only strictly normal crossings.

Proof. Necessity has been proven by Lemma 5. Hence it is sufficient to show sufficiency. Let $q \in f(X)$ and $\left\{p_{1}, \ldots, p_{r}\right\}=f^{-1}(q)$. As in the proof of Theorem 3.11 in $\left[3\right.$, p. 85], there exist a neighborhood $W_{q}$ of $q$ in $Y$ and neighborhoods $U_{i}$ of $p_{i}$ in $X(1 \leq i \leq r)$ which satisfy

(1) $U_{i} \cap U_{j}=\emptyset(1 \leq i, j \leq r$ for $i \neq j)$,

(2) $f \mid U_{i}$ is a $1: 1$ proper immersion into $W_{q}$,

(3) $f\left(U_{i}\right) \subset W_{q} \subset \overline{W_{q}} \subset \operatorname{Int} Y$,

(4) $f^{-1}\left(W_{q}\right)=\bigcup_{i=1}^{r} U_{i}$

(5) $W_{q}$ can be chosen as small as desired.

Then put $Y_{i}=f\left(U_{i}\right)(1 \leq i \leq r)$. By using the arguments in [3], we know that $Y_{1}, \ldots, Y_{r}$ are submanifolds with or without boundary and assume that only $Y_{i}(1 \leq i \leq k)$ have non-empty boundary for some $k(\leq r)$. Then the assumption of strictly normal crossings implies that $\partial Y_{i}(1 \leq i \leq k)$ and $Y_{j}$ $(k+1 \leq j \leq r)$ are in general position at $q$. So, we may choose $W_{q}$ so that $Y_{i}(1 \leq i \leq k)$ are simultaneously linearized orthonormally in $W_{q}$ although our case permits to have boundary.

From the argument of a partition of unity, it is sufficient to prove that $f$ is strongly infinitesimally stable for vector fields along $f$ whose supports are in $f^{-1}\left(W_{q}\right)$. Let $t_{i}$ be $t_{i}=\omega \circ\left(f \mid U_{i}\right)^{-1}$ which is a compactly supported vector field on $Y_{i}$, where $\omega \in C_{f}^{\infty}(X, T Y)$ with supp $\omega \subset f^{-1}\left(W_{q}\right)$. Then we show that there exists a vector field $t$ on $W_{q}$ so that $t \mid Y_{i}-t_{i}$ is tangential to $Y_{i}$ and to $\partial Y_{i}$ on $\partial Y_{i}$ for any $i$. 
$Y_{1}, \ldots, Y_{r}$ will be regarded to be subspaces in $\mathbf{R}^{n}$ with admitting to have boundary. To avoid complexity, we may assume $Y_{i}$ have boundary and $Y_{i}=\widetilde{Y}_{i}^{\partial}(1 \leq i \leq r)$ for some $m$-dimensional vector spaces $\tilde{Y}_{i}$ with bases in $\mathbf{R}^{n}$. By using the arguments of Lemma 3.8 in [3, p. 84], we can construct a vector field $t$ such that $t \mid Y_{i}-t_{i}$ is tangential to $Y_{i}$.

Based on this $t$, we will construct a new vector field $t^{\partial}$ on $W_{q}$ so that $t^{\partial} \mid Y_{i}-t_{i}$ is tangential to $Y_{i}$ on $Y_{i}$ and to $\partial Y_{i}$ on $\partial Y_{i}$. For the case of $r=1$, it is easy to find a vector field $v_{1}$ on $W_{q}$ whose elements are tangential to $Y_{1}$ so that $\left(t+v_{1}\right) \mid Y_{1}-t_{1}$ is tangential to $Y_{1}$ on $Y_{1}$ and to $\partial Y_{1}$ on $\partial Y_{1}$. For the case of $r>1$, we can find a vector field $v_{i}$ on $W_{q}$ whose elements are tangential to $Y_{i}$ and orthogonal to $\partial Y_{i}$ so that $\left(t+v_{i}\right) \mid Y_{i}-t_{i}$ is tangential to $Y_{i}$ on $Y_{i}$ and to $\partial Y_{i}$ on $\partial Y_{i}$. Then by Lemma 3.7 of $[3$, p. 83], our condition of strictly normal crossings means that the elements of $v_{i}$ can be assumed to be tangential to $Y_{j}$ on $Y_{j}$ and to $\partial Y_{j}$ on $\partial Y_{j}$ for $i \neq j$. Hence it is sufficient to define $t^{\partial}$ by $t^{\partial}=t+\left(v_{1}+\cdots+v_{r}\right)$, since $t^{\partial} \mid Y_{i}-t_{i}=(t+$ $\left.v_{i}\right) \mid Y_{i}-t_{i}+\left(v_{1}+\cdots+v_{i-1}+v_{i+1}+\cdots+v_{r}\right)$. Extend $t^{\partial}$ to a vector field on $Y$ such as $t^{\partial} \equiv 0$ off $W_{q}$. Then $\omega^{\partial}=\omega-t^{\partial} \circ f$ satisfies that for $p \in U_{i}, \omega^{\partial}(p)=\omega(p)-t^{\partial} \circ(f(p))$ is tangent to $f\left(U_{i}\right)$ at $f(p)$ and that for $p^{\prime} \in \partial U_{i}, \omega^{\partial}\left(p^{\prime}\right)$ is tangent to $f\left(\partial U_{i}\right)=\partial Y_{i}$ at $f\left(p^{\prime}\right)$. Therefore there exists a unique vector field $s_{i}^{\partial}$ on $U_{i}$ such that $s_{i}^{\partial} \mid \partial U_{i}$ is tangential to $\partial U_{i}$ and that $(d f) \circ s_{i}^{\partial}=\omega^{\partial}$ holds. The remainder of our proof is similar to that of Theorem 3.11 of $\left[3\right.$, p. 85]. Finally we obtain a vector field $s^{\partial} \in C^{\infty}(T X)$ so that $s^{\partial} \mid\left(f^{-1}\left(W_{q}\right) \cap \partial X\right) \in C^{\infty}\left(T\left(f^{-1}\left(W_{q}\right) \cap \partial X\right)\right), s^{\partial}=0$ off $f^{-1}\left(W_{q}\right)$ and $\omega=(d f) \circ s^{\partial}+t^{\partial} \circ f$ are satisfied.

Proposition 2 Let $\operatorname{dim} Y=2 \cdot \operatorname{dim} X$. Then $f: X \rightarrow Y$ is stable if and only if $f$ is an immersion with strictly normal crossings and satisfies $f(X) \subset \operatorname{Int} Y$.

Proof. It is an easy modification of the argument in the proof of Proposition 3.12 in $[3$, p. 86] to the case with boundary. Sufficiency has been proven. By Lemma 2 the immersions of $X$ into $Y$ are open and dense, hence every stable map $X \rightarrow Y$ must be an immersion, then Corollary 1 implies that any stable map is an immersion with strictly normal crossings, and $f(X) \subset$ Int $Y$ is obtained by the same reason stated in the proof of Lemma 5 . 


\section{Characterization of fold maps on manifolds with boundary}

In this section, we prove our main result as Theorem 1 which clarifies the structure of fold maps $X \rightarrow Y(m \geq n) . X$ and $Y$ are same with that in $\S 3$.

Remark 1 Let $f: X \rightarrow Y$ be a submersion with $S_{1,0}$, then $f \mid S_{1,0}(f)$ is an immersion.

Definition 6 (Global condition $(\mathrm{G})$ ) Let $f: X \rightarrow Y$ be a submersion with $S_{1,0}$ so that $f \mid \partial X$ is stable. Since $f \mid \partial X$ is a Boardman map, $f \mid \partial X$ has the Thom-Boardman stratification. Then it is known that on every stratum $S_{r_{1}, \ldots, r_{s}, 0}(f \mid \partial X), f \mid S_{r_{1}, \ldots, r_{s}, 0}(f \mid \partial X): S_{r_{1}, \ldots, r_{s}, 0}(f \mid \partial X) \rightarrow Y$ is an immersion. We consider the tangent spaces on $S_{1,0}(f)$ as those on $S_{1,0}(\tilde{f})$ with the extended manifolds $\widetilde{X}$ and $\widetilde{Y}$ in the sense of Definition 1 and define a global condition $(\mathrm{G})$ as follows:

Case 1: When $\operatorname{dim} X=\operatorname{dim} Y$, for any $q \in Y$ put a finite set $f^{-1}(q) \cap$ $\left(\partial X \cup S_{1,0}(f)\right)=\left\{p_{1}, \ldots, p_{N}\right\}$ so that $\partial X \backslash S_{1,0}(f) \ni p_{1}, \ldots, p_{N_{1}}, S_{1,0}(f) \backslash$ $\partial X \ni p_{N_{1}+1}, \ldots, p_{N_{1}+N_{2}}$ and $S_{1,0}(f) \cap \partial X \ni p_{N_{1}+N_{2}+1}, \ldots, p_{N_{1}+N_{2}+N_{3}}=$ $p_{N}$. Then $d(f \mid \partial X)_{p_{i}}\left(T_{p_{i}} \partial X\right), d\left(f \mid S_{1,0}(f)\right)_{p_{j}}\left(T_{p_{j}} S_{1,0}(f)\right)$ and $d\left(f \mid S_{1,0}(f) \cap\right.$ $\partial X)_{p_{k}}\left(T_{p_{k}}\left(S_{1,0}(f) \cap \partial X\right)\right)\left(1 \leq i \leq N_{1}, N_{1}+1 \leq j \leq N_{1}+N_{2}, N_{1}+N_{2}+\right.$ $\left.1 \leq k \leq N_{1}+N_{2}+N_{3}\right)$ are in general position at $q$.

Case 2: When $\operatorname{dim} X \geq \operatorname{dim} Y+1$, for any $q \in Y, f^{-1}(q) \cap(S(f \mid \partial X) \cup$ $\left.S_{1,0}(f)\right)$ is a set of discrete points in a compact set $X$, so this is a finite set, hence put $f^{-1}(q) \cap\left(S(f \mid \partial X) \cup S_{1,0}(f)\right)=\left\{p_{1}, \ldots, p_{N}\right\}$ so that $S(f \mid \partial X) \backslash$ $S_{1,0}(f) \ni p_{1}, \ldots, p_{N_{1}}, S_{1,0}(f) \backslash S(f \mid \partial X) \ni p_{N_{1}+1}, \ldots, p_{N_{1}+N_{2}}$ and $S_{1,0}(f) \cap$ $S(f \mid \partial X) \ni p_{N_{1}+N_{2}+1}, \ldots, p_{N_{1}+N_{2}+N_{3}}=p_{N}$. Then

$d\left(f \mid S_{r_{1}, \ldots, r_{s(i)}, 0}(f \mid \partial X)\right)_{p_{i}}\left(T_{p_{i}} S_{r_{1}, \ldots, r_{s(i)}, 0}(f \mid \partial X)\right), d\left(f \mid S_{1,0}(f)\right)_{p_{j}}\left(T_{p_{j}} S_{1,0}(f)\right)$ and $d(f \mid \partial X)_{p_{k}}\left(T_{p_{k}}\left(S_{1,0}(f) \cap \partial X\right)\right) \cap d(f \mid \partial X)_{p_{k}}\left(T_{p_{k}} S_{t_{1}, \ldots, t_{s(k)}, 0}(f \mid \partial X)\right)$ $\left(1 \leq i \leq N_{1}, N_{1}+1 \leq j \leq N_{1}+N_{2}, N_{1}+N_{2}+1 \leq k \leq N_{1}+N_{2}+N_{3}\right)$ are in general position at $q$, where $S_{r_{1}, \ldots, r_{s(i)}, 0}(f \mid \partial X)$ (or $\left.S_{t_{1}, \ldots, t_{s(k)}, 0}(f \mid \partial X)\right)$ is the stratum of $f \mid \partial X$ containing $p_{i}$ (resp. $p_{k}$ ) and of course $S_{0}(f \mid \partial X)$ means $\partial X \backslash S(f \mid \partial X)$.

Theorem 1 Let $f: X \rightarrow Y$ be a submersion with $S_{1,0}$. Then $f$ is stable if and only if $f$ satisfies the following conditions (1), (2) and (3):

(1) Every point in $S_{1,0}(f)$ is a fold point.

(2) $f \mid \partial X$ is stable and $f$ satisfies the condition (G). 
(3) $f(X) \subset \operatorname{Int} Y$.

Proof. Necessity: First, we show (3). If $f(X) \cap \partial Y \neq \emptyset$, then a slight perturbation of $f$ is possible to change $f$ such as $f(X) \cap \partial Y=\emptyset$, and then, the diffeomorphism type of $f$ is not kept. This implies (3).

Second, we show (2). The stability of $f \mid \partial X$ is already shown by Corollary 2. Next, the definition of stability implies that there exists an open neighborhood $N(f)$ of $f$ in $C^{\infty}(X, Y)$ such that every $g$ in $N(f)$ satisfies $g=\varphi \circ f \circ \phi^{-1}$ where $\phi: X \rightarrow X$ and $\varphi: Y \rightarrow Y$ are some diffeomorphisms. Then $\operatorname{rank}(d f)_{p}=\operatorname{rank} d\left(\varphi \circ f \circ \phi^{-1}\right)_{\phi(p)}(p \in X)$ holds, thus $\phi\left(S_{1,0}(f)\right)=$ $S_{1,0}(g)$ holds, i.e., the right-left equivalence preserves $S_{1,0}(f)$. Similarly, the right-left equivalence preserves the Boardman sets $S_{r_{1}, \ldots, r_{s}, 0}(f \mid \partial X)$ also. Hence for each case 1 or 2 , if the condition $(G)$ is not hold then a slight perturbation of $f$ is possible to change the diffeomorphism type of $f$, i.e., $f$ is not stable. More precisely, we can use the arguments similar to the proof of Proposition 5.6 in [3, p. 158].

Third, we show (1). We suppose that $S_{1,0}(f)$ intersects not transversely with $\partial X$ at $p$ in $X$. From the definition of $S_{1,0}(f)$, we can express $\tilde{f}$ as $\left(x_{1}, \ldots, x_{m}\right) \mapsto\left(x_{1}, \ldots, x_{n-1}, x_{n}{ }^{2} \pm \cdots \pm x_{m}{ }^{2}\right)$ around $p$ in $\tilde{X}$. Since $S_{1,0}(f)$ corresponds to $\left\{x_{n}=x_{n+1}=\cdots=x_{m}=0\right\}$, we may assume that $\partial X$ corresponds to $\left\{x_{m}=\phi\left(x_{1}, x_{2}, \ldots, x_{m-1}\right)\right\}$ with a $C^{\infty}$-function $\phi$ satisfying $\frac{\partial \phi}{\partial x_{m}}(0) \neq 0, \frac{\partial \phi}{\partial x_{1}}(0)=\cdots=\frac{\partial \phi}{\partial x_{m-1}}(0)=0$, and then we may assume that Int $X$ corresponds to $\left\{x_{m}>\phi\left(x_{1}, x_{2}, \ldots, x_{m-1}\right)\right\}$.

We put $\tau \in C_{f}^{\infty}(X, T Y)$ by

$$
\tau\left(x_{1}, \ldots, x_{m}\right)=\left(f\left(x_{1}, \ldots, x_{m}\right) ;(\underbrace{0, \ldots, 0}_{n-1}, 2 x_{m})\right) .
$$

Then we will show that if $\tau$ is expressed by $\tau=d f \circ \zeta^{\partial}+\eta^{\partial} \circ f \cdots(\star)$ where $\zeta^{\partial}=\left(\left(x_{1}, \ldots, x_{m}\right) ;\left(w_{1}, \ldots, w_{m}\right)\right) \in C^{\infty}(T X)$ with $\zeta^{\partial} \mid \partial X \in C^{\infty}(T \partial X)$ and $\eta^{\partial}=\left(\left(y_{1}, \ldots, y_{n}\right) ;\left(v_{1}, \ldots, v_{n}\right)\right) \in C^{\infty}(T Y)$ with $\eta^{\partial} \mid \partial Y \in C^{\infty}(T \partial Y)$, then this produces a contradiction. $(\star)$ means $w_{k}+v_{k}\left(x_{1}, \ldots, x_{n-1}, x_{n}{ }^{2} \pm\right.$ $\left.\cdots \pm x_{m}^{2}\right)=0(1 \leq k \leq n-1), 2 x_{n} w_{n} \pm 2 x_{n+1} w_{n+1} \pm \cdots \pm 2 x_{m} w_{m}+$ $v_{n}\left(x_{1}, \ldots, x_{n-1}, x_{n}{ }^{2} \pm \cdots \pm x_{m}^{2}\right)=2 x_{m}$. Then the partial differential $2 x_{n} \frac{\partial w_{n}}{\partial x_{m}} \pm \cdots \pm 2 x_{m} \frac{\partial w_{m}}{\partial x_{m}} \pm 2 w_{m} \pm 2 x_{m} \frac{\partial v_{n}}{\partial x_{m}}=2$ implies $w_{m}(0) \neq 0$. Therefore $\zeta^{\partial}$ is not tangential to $\partial X$ at $p$. This is a contradiction.

Before proving sufficiency, we prepare the following Lemma 7. 
Lemma 7 Let $f: X \rightarrow Y$ be a submersion with $S_{1,0}(f)$, and suppose that the conditions (1) and (3) of Theorem 1 are satisfied. Then, for $p \in S_{1,0}(f) \cap$ $\partial X$, there exist local coordinates $t, x_{2}, \ldots, x_{m}$ centered at $p, y_{1}, \ldots, y_{n}$ centered at $f(p)$ and $\phi$ a $C^{\infty}$-function of $x_{2}, \ldots, x_{m}$ such that $f$ is expressed by

$$
\begin{aligned}
& \left(t, x_{2}, \ldots, x_{m}\right) \\
& \quad \mapsto\left(t+\phi\left(x_{2}, \ldots, x_{m}\right), x_{2}, \ldots, x_{n-1}, x_{n}{ }^{2} \pm x_{n+1}{ }^{2} \pm \cdots \pm x_{m}{ }^{2}\right),
\end{aligned}
$$

where $\partial X$ corresponds to $\{t=0\}$ and Int $X$ corresponds to $\{t>0\}$.

Proof. Theorem 4.5 in $[3$, p. 88$]$ has shown that in the previous extended manifolds $\widetilde{X} \supset X$ and $\tilde{Y} \supset Y$ such as $p \in \operatorname{Int} \tilde{X}$ with an extended $C^{\infty}$. function $\tilde{f}$ of $f, \tilde{f}$ is expressed by $\left(x_{1}, \ldots, x_{m}\right) \mapsto\left(x_{1}, \ldots, x_{n-1}, x_{n}{ }^{2} \pm \cdots \pm\right.$ $x_{m}^{2}$ ) around a neighborhood $U_{p}$ of $p$, and a neighborhood $V_{\tilde{f}(p)}$ of $\tilde{f}(p)$, where $S_{1,0}(\tilde{f})$ corresponds to $x_{n}=x_{n+1}=\cdots=x_{m}=0$.

Since the codimension of $\partial X$ is one, by the condition (1) we may assume that $\partial X$ is a graph of $x_{2}, \ldots, x_{m}$ in $\widetilde{X}$ for $x_{1}=\phi\left(x_{2}, \ldots, x_{m}\right)$ with a $C^{\infty}$-function $\phi$. Through a change of coordinates such as $x_{1}^{\prime}=x_{1}$ $\phi\left(x_{2}, \ldots, x_{m}\right), x_{2}^{\prime}=x_{2}, \ldots, x_{m}^{\prime}=x_{m}$, we can write $\tilde{f}$ by $\left(x_{1}^{\prime}, \ldots, x_{m}^{\prime}\right) \mapsto$ $\left(x_{1}^{\prime}+\phi\left(x_{2}^{\prime}, \ldots, x_{m}^{\prime}\right), x_{2}^{\prime}, \ldots, x_{n-1}^{\prime}, x_{n}^{\prime 2} \pm x_{n+1}^{\prime}{ }^{2} \pm \cdots \pm x_{m}^{\prime}{ }^{2}\right)$ where $\left\{x_{1}^{\prime}=0\right\}$ corresponds to $\partial X$ and we may assume $\left\{x_{1}^{\prime}>0\right\}$ corresponds to Int $X$. This induces our conclusion.

Remark 2 By calculating the rank of Jacobian matrix of $f$ based on Lemma 7, we know that $S_{1,0}(f) \cap \partial X \subset S(f \mid \partial X)$.

Proof of the sufficiency of Theorem 1. We use the notion of stability appeared in the proof of Lemma 6, i.e.; we will show that if $f$ satisfies the conditions (1), (2) and (3) then for every $v$ with $1 \leq v \leq n+1$ and any diagonal element $\sigma$ in $J_{v, 0}^{n}(X, Y), j_{v, 0}^{n} f$ is transverse to $\mathcal{D}_{\sigma}^{v}$.

From the arguments in the case of manifolds without boundary, if the source of $\sigma$ does not contain a point on $\partial X, j_{v, 0}^{n} f$ is transverse to $\mathcal{D}_{\sigma}^{v}$. Therefore, we may assume that the source of $\sigma$ contains a point of $\partial X$. We give a proof by two steps.

Step 1. [If $f$ satisfies the conditions (1), (2) and (3) then it will be shown that there exists a sufficiently small neighborhood $N$ of $f$ in $C^{\infty}(X, Y)$ such that for any $\tilde{g}$ in $D(\tilde{f})=\left\{\left.\tilde{h} \in C^{\infty}(\tilde{X}, \tilde{Y})\right|^{\exists} \tilde{\varphi} \circ \tilde{f} \circ \circ^{\exists} \tilde{\phi}^{-1}=\tilde{h}\right\} \cap N$ with diffeomorphisms $\tilde{\phi}: \widetilde{X} \rightarrow \widetilde{X}$ and $\tilde{\varphi}: \widetilde{Y} \rightarrow \tilde{Y}$, two maps $f=\tilde{f} \mid X$ and 
$g=\tilde{g} \mid X$ are R-L (i.e., right-left) equivalent.]

Indeed, the global condition $(\mathrm{G})$, the condition of transversality between $S_{1,0}(f)$ and $\partial X$, and $f(X) \subset$ Int $Y$ guarantee the existence of the following diffeomorphism $\tilde{\phi}^{\prime}: \widetilde{X} \rightarrow \widetilde{X}$ which satisfies from (i) to (iii). (See Figure 1.)

(i) $\tilde{\phi}^{\prime-1} \circ \tilde{\phi}^{-1}(X)=X\left(\tilde{\phi}^{\prime-1} \circ \tilde{\phi}^{-1}(\partial X)=\partial X\right.$ automatically. $)$

(ii) $\tilde{\phi}^{\prime-1} \circ \tilde{\phi}^{-1}\left(S_{r_{1}, \ldots, r_{s}, 0}(g \mid \partial X)\right)=S_{r_{1}, \ldots, r_{s}, 0}(f \mid \partial X)$

(iii) If $p_{1}, p_{2} \in \tilde{X}$ satisfies $\tilde{f} \circ \tilde{\phi}^{-1}\left(p_{1}\right)=\tilde{f} \circ \tilde{\phi}^{-1}\left(p_{2}\right)$ then $\tilde{f} \circ \tilde{\phi}^{\prime-1} \circ \tilde{\phi}^{-1}\left(p_{1}\right)=$ $\tilde{f} \circ \tilde{\phi}^{\prime-1} \circ \tilde{\phi}^{-1}\left(p_{2}\right)$ is also satisfied. Oppositely, if $\tilde{f} \circ \tilde{\phi}^{-1}\left(p_{1}\right) \neq \tilde{f} \circ \tilde{\phi}^{-1}\left(p_{2}\right)$ holds then $\tilde{f} \circ \tilde{\phi}^{\prime-1} \circ \tilde{\phi}^{-1}\left(p_{1}\right) \neq \tilde{f} \circ \tilde{\phi}^{\prime-1} \circ \tilde{\phi}^{-1}\left(p_{2}\right)$ is also satisfied.

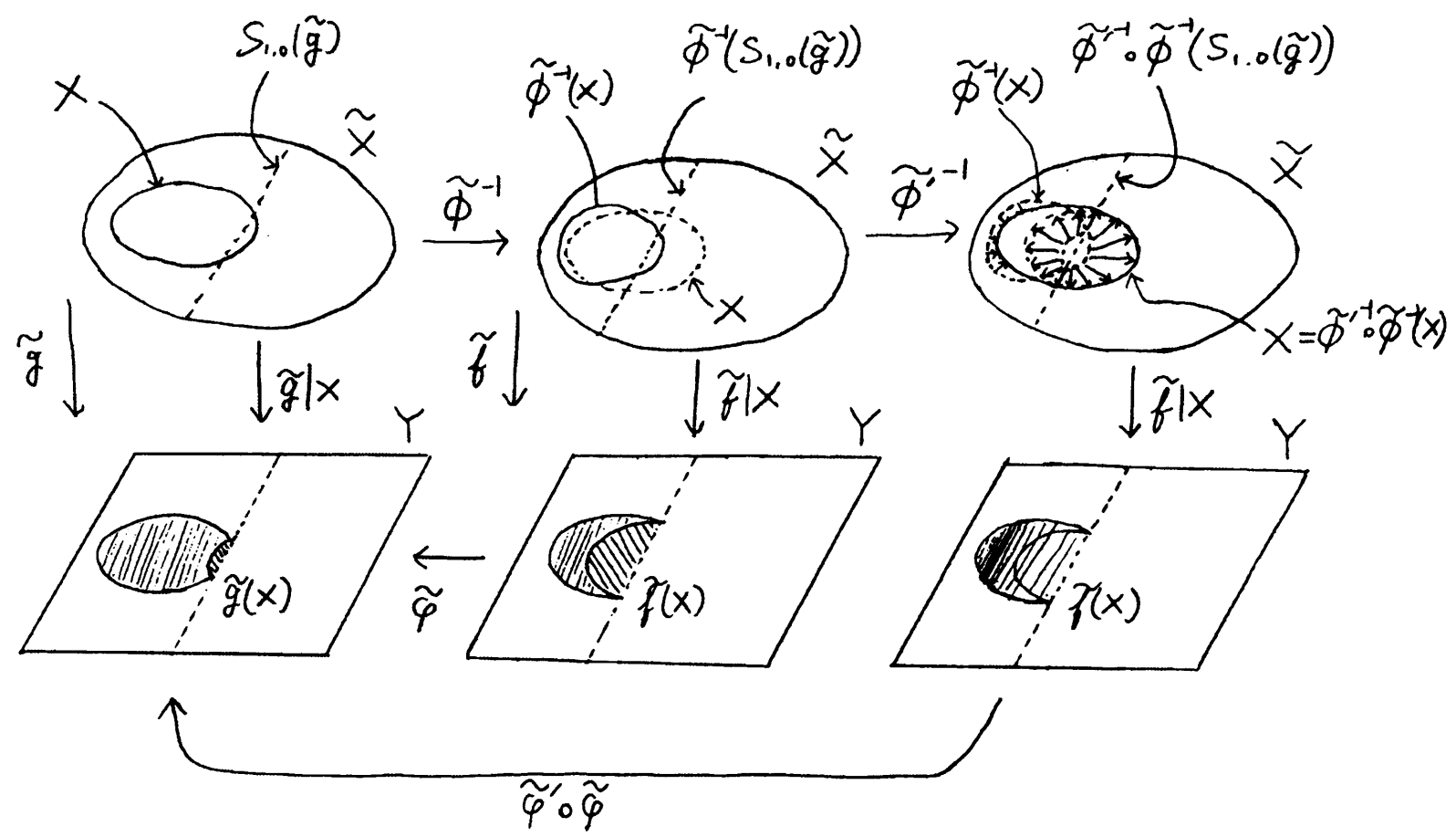

Fig. 1.

By the assumption of sufficiency, there exist diffeomorphisms $\phi^{\partial}: \partial X \rightarrow$ $\partial X$ and $\varphi^{\partial}: Y \rightarrow Y$ such that $\varphi^{\partial} \circ(\tilde{f} \mid \partial X) \circ\left(\phi^{\partial}\right)^{-1}=\tilde{g} \mid \partial X$. Here we extend $\varphi^{\partial}$ to a diffeomorphism $\tilde{\varphi^{\partial}}: \tilde{Y} \rightarrow \tilde{Y}$ with holding $\tilde{\varphi^{\partial}} \mid Y=\varphi^{\partial}$. Thus we may assume that the previous $\tilde{\phi}^{\prime}$ satisfies $\left(\tilde{\phi}^{\prime-1} \circ \tilde{\phi}^{-1}\right) \mid \partial X=\left(\phi^{\partial}\right)^{-1}$. With respect to the above $\tilde{\phi}^{\prime}$, we can find a diffeomorphism $\tilde{\varphi}^{\prime}: \widetilde{Y} \rightarrow \tilde{Y}$ so that $\tilde{\varphi}^{\prime} \circ \tilde{\varphi} \circ \tilde{f} \circ \tilde{\phi}^{\prime-1} \circ \tilde{\phi}^{-1}=\tilde{g}$ and $\tilde{\varphi}^{\prime} \circ \tilde{\varphi}(Y)=Y$ are satisfied, because $\tilde{\varphi}^{\partial} \circ$ $\tilde{\varphi}^{-1}: \tilde{Y} \rightarrow \tilde{Y}$ is a diffeomorphism such as $\left(\tilde{\varphi}^{\partial} \circ \tilde{\varphi}^{-1}\right) \circ \tilde{\varphi}=\tilde{\varphi}^{\partial}$. Thus we can $\operatorname{deform}\left(\tilde{\varphi}^{\prime} \stackrel{\text { def }}{=}\right) \tilde{\varphi}^{\partial} \circ \tilde{\varphi}^{-1}$ outside $\tilde{\varphi} \circ \tilde{f}(\partial X)$ so that $\tilde{\varphi}^{\prime} \circ \tilde{\varphi} \circ \tilde{f} \circ \tilde{\phi}^{\prime-1} \circ \tilde{\phi}^{-1}=\tilde{g}$ 
holds. Then $\left(\tilde{\varphi}^{\prime} \circ \tilde{\varphi} \mid Y\right) \circ f \circ\left(\tilde{\phi} \circ \tilde{\phi}^{\prime} \mid X\right)^{-1}=g$ gives a R-L equivalence between $f$ and $g$.

Step 2. [We will show that, if $f: X \rightarrow Y$ is not stable then all of the previous conditions (1), (2) and (3) are not satisfied, i.e., the non-existence of $N$ in the claim of Step 1 follows.]

It is sufficient to prove that if there is a diagonal element $\sigma$ in $J_{v, 0}^{n}(X, Y)$ for a $v(1 \leq v \leq n+1)$ so that $j_{v, 0}^{n} f$ is not transverse to $\mathcal{D}_{\sigma}^{v}$, then for any neighborhood $N^{\prime}$ of $f$ in $C^{\infty}(X, Y)$, there exists a $g^{\prime}$ in $D(\tilde{f}) \cap N^{\prime}$ which does not have definitely diffeomorphisms $\phi: X \rightarrow X$ and $\varphi: Y \rightarrow Y$ such as $\varphi \circ f \circ \phi^{-1}=g^{\prime}$.

We will explain that for the above any $N^{\prime}$, there exists such a $g^{\prime}$ by separating the assumption into the following two cases.

Case 1: $\quad\left[\sigma\right.$ is an isolated point in $j_{v, 0}^{n} f \cap \mathcal{D}_{\sigma}^{v}$. $]$

Let $\tilde{X}, \widetilde{Y}$ be extended manifolds of $X$ and $Y$ respectively due to the definition of $S_{1,0}(f)$. First, we can regard that $j_{v, 0}^{n} f$ is in $j_{v, 0}^{n} \tilde{f}$ and $\mathcal{D}_{\sigma}^{v}$ is in $J_{v, 0}^{n}(\tilde{X}, \tilde{Y})$ by $J_{v, 0}^{n}(X, Y) \subset J_{v, 0}^{n}(\tilde{X}, \tilde{Y})$. For an appropriate manifold $X_{-}$ diffeomorphic to $X$ and an appropriate diffeomorphism $\tilde{\phi}: \tilde{X} \rightarrow \tilde{X}$ such as $X_{-} \subset \operatorname{Int} X \subset X \subset \tilde{X}$ and $\tilde{\phi}\left(X_{-}\right)=X$, put $\tilde{g}=\operatorname{id}_{\tilde{Y}} \circ \tilde{f} \circ \tilde{\phi}^{-1}$ where $\operatorname{id}_{\widetilde{Y}}: \widetilde{Y} \rightarrow \tilde{Y}$ is the identity, and then we may assume that $\tilde{g}$ is sufficiently close to $\tilde{f}$. Then we show that $f$ and $g=\tilde{g} \mid X$ are not R-L equivalent. If $f$ and $g$ are R-L equivalent then there must exist diffeomorphisms $\phi$ : $X \rightarrow X$ and $\varphi: Y \rightarrow Y$ such as $g=\varphi \circ f \circ \phi^{-1}$. However, by taking an

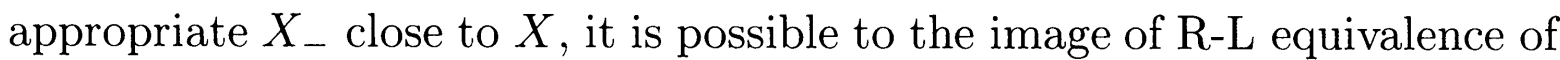
$\sigma, j_{v, 0}^{n} \operatorname{id}_{Y} \circ \sigma \circ j_{v, 0}^{n} \tilde{\phi}^{-1}$, is not in $\mathcal{D}_{\sigma}^{v}$ from the assumption of Case 1 . Hence it is shown that $f$ and $g$ are not R-L equivalent.

Case 2: $\quad\left[\sigma\right.$ is not an isolated point in $j_{v, 0}^{n} f \cap \mathcal{D}_{\sigma}^{v}$. $]$

Let $\tilde{X}, \widetilde{Y}$ be extended manifolds of $X$ and $Y$ respectively due to the definition of $S_{1,0}(f)$. Then the non-transversality condition between $j_{v, 0}^{n} f$ and $\mathcal{D}_{\sigma}^{v}$ admits to the existences of $\tilde{f}$ with $\tilde{X}, \tilde{Y}$ and a neighborhood of $\sigma$ in $J_{v, 0}^{n}(\tilde{X}, \tilde{Y}), N(\sigma)$, so that $\left(j_{v, 0}^{n} \tilde{f} \backslash j_{v, 0}^{n} f\right) \cap \mathcal{D}_{\sigma}^{v} \cap N(\sigma)=\emptyset$ is satisfied, or so that for any neighborhood of $\sigma$ in $N(\sigma), N^{\prime}(\sigma)(\subset N(\sigma)),\left(j_{v, 0}^{n} \tilde{f} \backslash j_{v, 0}^{n} f\right) \cap$ $\mathcal{D}_{\sigma}^{v} \cap N^{\prime}(\sigma)$ has more than two components in $J_{v, 0}^{n}(\tilde{X}, \tilde{Y})$ (i.e., it seems to be ramified.), where $\mathcal{D}_{\sigma}^{v}$ is defined in the subspace $J_{v, 0}^{n}(X, Y) \subset J_{v, 0}^{n}(\widetilde{X}, \widetilde{Y})$. 
For an appropriate manifold $X_{+}$diffeomorphic to $X$ and an appropriate diffeomorphism $\tilde{\phi}: \widetilde{X} \rightarrow \widetilde{X}$ such as $X \subset \operatorname{Int} X_{+} \subset X_{+} \subset$ Int $\widetilde{X} \subset \widetilde{X}$ and

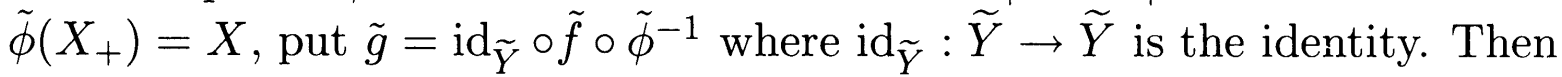
we may assume that $\tilde{g}$ is sufficiently close to $\tilde{f}$, and then we show that $f$ and $g=\tilde{g} \mid X$ are not R-L equivalent. Indeed, since a diffeomorphism on $X$ induces a diffeomorphism of $\partial X$ onto $\partial X$, if $f$ and $g$ are R-L equivalent by $g=\varphi \circ f \circ \phi^{-1}$ with some diffeomorphisms $\phi: X \rightarrow X$ and $\varphi: Y \rightarrow Y$, then the above induced R-L equivalence on $\partial X$ must map the point $\sigma$ into an empty set or at least two distinct points from the assumption of Case 2 . This is a contradiction.

\section{Appendix}

We give an appendix as the following Lemma 8. This will be welcomed for reader who requests for severe explanations in the proof of Theorem 1 .

Lemma 8 Let $Y$ be a manifold with submanifolds $Y_{1}, \ldots, Y_{r}$. Suppose $q \in Y_{1} \cap \cdots \cap Y_{r}$ and $Y_{1}, \ldots, Y_{r}$ are in general position at $q$. Then, for diffeomorphisms $f_{i}: Y_{i} \rightarrow f_{i}\left(Y_{i}\right) \subset Y$ close to the identity $\operatorname{id}_{Y_{i}}: Y_{i} \rightarrow Y_{i} \subset$ $Y$ with the Whitney $C^{\infty}$-topology, there exists a diffeomorphism $\Phi: Y \rightarrow Y$ which satisfy $\Phi\left(Y_{1} \cup \cdots \cup Y_{r} \cap N\right)=f_{1}\left(Y_{1}\right) \cup \cdots \cup f_{r}\left(Y_{r}\right) \cap \Phi(N)$ and $\Phi\left(Y_{i} \cap\right.$ $N)=f_{i}\left(Y_{i}\right) \cap \Phi(N)(1 \leq i \leq r)$ for a neighborhood of $q$ in $Y, N$.

Proof. (sketch) First, we can find a diffeomorphism $\widetilde{\Phi}: \mathbf{R}^{\operatorname{dim} Y}(\supset N) \rightarrow$ $\mathbf{R}^{\operatorname{dim} Y}$ and $q^{\prime} \in Y$ such that $\widetilde{\Phi}\left(T_{q} Y_{1} \cup \cdots \cup T_{q} Y_{r} \cap N\right)=T_{q^{\prime}} f_{1}\left(Y_{1}\right) \cup \cdots \cup$ $T_{q^{\prime}} f_{r}\left(Y_{r}\right) \cap \widetilde{\Phi}(N)$ with $\widetilde{\Phi}\left(T_{q} Y_{i} \cap N\right)=T_{q^{\prime}} f_{i}\left(Y_{i}\right) \cap \widetilde{\Phi}(N)(1 \leq i \leq r)$.

Next consider a deformation by diffeomorphisms $\widetilde{\Phi}_{1}, \widetilde{\Phi}_{2}: \mathbf{R}^{\operatorname{dim} Y}(\supset$ $N) \rightarrow \mathbf{R}^{\operatorname{dim} Y}$ so that $\widetilde{\Phi}_{1}\left(T_{q} Y_{i}\right) \cap N=Y_{i} \cap N$ with $\widetilde{\Phi}_{1}(q)=q$ and $\widetilde{\Phi}_{2}\left(T_{q^{\prime}} f_{i}\left(Y_{i}\right)\right) \cap \widetilde{\Phi}(N)=f_{i}\left(Y_{i}\right) \cap \widetilde{\Phi}(N)$ with $\widetilde{\Phi}_{2}\left(q^{\prime}\right)=q^{\prime}$ (for $\left.1 \leq i \leq r\right)$.

Problem 1 For the stable maps treated in this paper, consider the corresponding theorem with that in Levine [4, p. 2567].

Acknowledgements The author would like to express his sincere gratitude to Professor O. Saeki for his helpful comments after careful reading and to Professor T. Ohmoto for useful discussions with him at Kyoto University. 


\section{References}

[1] Boardman J.M., Singularities of differential maps. Publ. Math. I.H.E.S. 33 (1967), 21-57.

[2] Essen Erik von, On stability of $C^{\infty}$ mappings of manifolds with boundary. Math. Scand. 43 (1978), 197-203.

[3] Golubitsky M. and Guillemin V., Stable mappings and their singularities. Graduate Texts in Math. 14, Springer-Verlag (1973).

[4] Levine H., Computing the Euler characteristic of a manifold with boundary. Proc. Amer. Math. Soc. 123 (1995), 2563-2567.

[5] Mather J.N., Stability of $C^{\infty}$ mappings: II. Infinitesimal stability implies stability. Ann. of Math. 89 (1969), 254-291.

[6] Shibata N., Infinitesimal stability of stable maps on manifolds with boundary. Kumamoto J. of Math. 16 (2003), 43-49.

[7] Shibata N., On non-singular stable maps of 3-manifolds with boundary into the plane. Hiroshima Math. J. 30 (2000), 415-435.

1996-1 Misonou, Saijyō

Higashi-hiroshima 739-0024

Japan

E-mail: nshibata@wonder.ocn.ne.jp 